Research Article

\title{
Nonlinear Optimization Method for Transmission Error of Hypoid Gear Machined by the Duplex Helical Method
}

\author{
Shunxing Wu, ${ }^{1,2}$ Hong-Zhi Yan $\mathbb{D D}^{1}{ }^{1}$ Rengui Bi, ${ }^{2}$ Zhiyong Wang, ${ }^{3}$ and Pengfei Zhu' \\ ${ }^{1}$ State Key Laboratory for High Performance Complex Manufacturing, Central South University, Changsha 410083, China \\ ${ }^{2}$ College of Physics and Electromechanical Engineering, Jishou University, Jishou 416000, China \\ ${ }^{3}$ College of Mechanical and Electrical Engineering, Central South University of Forestry and Technology, Changsha 410004, China
}

Correspondence should be addressed to Hong-Zhi Yan; yhzcsu@163.com

Received 14 May 2020; Revised 16 October 2020; Accepted 11 November 2020; Published 29 November 2020

Academic Editor: Vasilios Spitas

Copyright (C) 2020 Shunxing Wu et al. This is an open access article distributed under the Creative Commons Attribution License, which permits unrestricted use, distribution, and reproduction in any medium, provided the original work is properly cited.

In this study, synchronous cutting of concave and convex surfaces for hypoid gear was achieved using a duplex helical method. Precise, nonlinear optimization of the transmission error driven by machine tool parameters was performed to reduce the vibration noise of the gear pair. First, the transmission error curve and contact path of the tooth surface of the initial pinion were solved using tooth contact analysis. Second, according to the preset parabolic transmission error curve, the initial gear was used to generate the target pinion, which coincided with the contact path of the initial pinion. Finally, a deviation correction model of the discrete points, corresponding to the contact paths on the concave and convex surfaces of the target and initial pinions, was established. This model was solved using the Levenberg-Marquard algorithm with the trust region strategy, to obtain optimized machine tool parameters. Synchronous optimization of the transmission errors of concave and convex surfaces of the pinion was achieved by correcting the deviations of the contact points. The effectiveness of the proposed method was verified by a numerical example and by performing a contact area rolling test.

\section{Introduction}

Owing to the strong bearing capacity and high structural reliability of hypoid gear pairs, they are widely used in transmission devices such as automobiles and helicopters. A hypoid gear is normally produced using a traditional cutting method (i.e., the five-cut method). In this approach, the vibration and noise can be controlled easily because the concave and convex surfaces are cut and modified separately $[1,2]$. Currently, the five-cut method is gradually being replaced by the duplex helical method. The duplex helical method is advantageous because of its higher machining efficiency and superior quality consistency compared to those of the five-cut method [3]. However, because the duplex helical method involves simultaneous processing of concave and convex surfaces, it is difficult to control the vibration and noise of the gear pair [4]. Owing to the increasing demand for comfort and reliability in cars, higher requirements are simultaneously being imposed for the vibration noise and life span of gear pairs. The vibration noise and life span of a gear pair are directly affected by the transmission error. Therefore, it is crucial to reduce the transmission error of gear pairs while ensuring efficient cutting in the duplex helical method $[5,6]$.

Presetting and optimizing the transmission error curve of a gear pair are effective ways to reduce vibration noise while improving service life. Researchers have investigated spiral bevel gear pairs cut using the five-cut method. Based on the local synthesis method, Litvin et al. [7] and Simon [8] reduced the transmission error amplitude of a gear pair by presetting the three second-order contact parameters of the transmission error curve. Taking the preset parameters of the local synthesis method as optimization variables, Wang et al. [9] and Wang et al. [10] optimized the load transmission error amplitude through a genetic algorithm and kriging algorithm, respectively. Considering that the local synthesis method can only control the contact performance near the reference point, Liu and Fan [11] proposed optimizing the transmission error amplitude based on the local synthesis method by taking the modified coefficient as the 
design variable. Zhuo et al. [5] optimized the transmission error amplitude through the genetic algorithm, which is constrained by the composite shape method. This optimization was accomplished using the normal curvature and short-range torsion of the tooth surface as the controlling parameters. By expressing the tooth surface as a higherorder polynomial, Artoni et al. [12] used the coefficient of the higher-order polynomial as the optimization variable. This approach was employed to optimize the loaded transmission error amplitude and the maximum contact stress. By expressing the machine tool parameters as a sixth-order polynomial on the cradle angle, Astoul et al. [13] reduced the amplitude of the transmission error by optimizing the polynomial coefficients.

Based on research, the transmission error amplitude can be reduced by optimizing the cutter head and machine tool parameters related to the transmission error. However, the transmission error amplitude cannot be obtained accurately. The target pinion tooth surface is obtained by presetting the transmission error on the pinion tooth surface, where it is a conjugate with the initial gear. The cutter head and machine tool parameters of the initial pinion can be modified by minimizing the deviation between the initial and the target pinions, which is a feasible method of obtaining the transmission error amplitude accurately. This modification process can be attributed to the least-squares optimization problem, which is a hot topic in current research. Krenzer [14] first proposed a linear regression correction method for machine tool parameters using a spiral bevel gear with minimum tooth surface deviation. Based on this research, Litvin et al. [15] and Stadtfeld [16] proposed minimizing tooth surface deviation as a nonlinear least squares optimization problem, and the accuracy of the machine tool parameters improved. Subsequently, to improve the stability and accuracy of the solution further, a sensitivity matrix describing the variations of the machine tool parameters relative to the tooth surface deviation was applied. The sequential quadratic programming algorithm $[17,18]$ and singular value decomposition algorithm [19] have also been used to correct the machine tool parameters. However, for the problem of excessive ill-health, the Hessian matrix has ill-health. In other words, the matrix is not positive, which makes accurate and stable solutions of nonlinear equations difficult to obtain. Therefore, the Levenberg-Marquard method $[20,21]$ is proposed to solve the correction value of the machine tool parameters. The core of the Levenberg-Marquard algorithm involves introducing a damping coefficient greater than zero to ensure that the Hessian matrix obtained in each iteration step is positive definite and reversible. Consequently, the iteration can be performed with high precision.

As previously stated, this approach optimizes the machining parameters related to the transmission error to reduce the transmission error amplitude or to correct the tooth surface deviation to obtain greater accurate transmission error amplitude. Limited research has been conducted on the duplex helical method. Unlike the five-cut method, the duplex helical method simultaneously cuts concave and convex surfaces. It is more difficult to obtain the transmission error amplitude accurately for a gear pair cut by the duplex helical method. Therefore, the high-precision optimization of the transmission error of a hypoid gear pair cut by the duplex helical method was investigated in this study.

\section{Tooth Surface Modeling of Hypoid Gear Pairs Machined by the Duplex Helical Method}

To optimize the transmission error of a gear pair, the machining mathematical model of a gear pair should first be established. In this study, the gear was machined by a nongenerated method and the pinion was machined by a duplex helical method. The detailed machining mathematical model of a gear is given in reference [7]. $R_{2}\left(u_{g}, \theta_{\mathrm{g}}\right)$ and $n_{2}\left(u_{g}, \theta_{g}\right)$ represent the tooth surface equation and unit normal vector of a gear in the gear coordinate system $S_{2}$, respectively, where $\left(u_{g}, \theta_{g}\right)$ are the Gaussian coordinates of the gear tooth surface. The detailed process of cutting a pinion is described below.

The coordinate systems applied for pinion generation are shown in Figure 1. $S_{m 1}, S_{c}$, and $S_{d}$ are coordinate systems rigidly fixed to the cutting machine. $S_{p}$ is a coordinate system rigidly fixed to the head cutter and rotates around the axis $z_{p}$. $S_{b}$ and $S_{1}$ are the movable coordinate systems rigidly fixed to the cradle and pinion, respectively. $S_{a}$ is the reference coordinate system of the tilt angle and swivel angle. When the pinion is cut by the duplex helical method, the cradle rotates around the axis $z_{m 1}$ while moving as a helix along the axis $z_{m 1}$. Simultaneously, the pinion makes a rotary motion around the axis $x_{d}$. The tooth surface of the pinion is cut into an envelope of the tool curved family through the generating movement. As shown in Figure 1, the machine tool settings $q_{1}, s_{r 1}, i, j, e_{1}, x_{b 1}, \gamma_{m 1}, x_{g 1}$, and $h l$ represent the center roll position, radial distance, tilt angle, swivel angle, work offset, sliding base, machine root angle, the machine center to the cross point, and the velocity coefficient of helical motion, respectively. $\varphi$ is the rotation angle of the pinion, $\varphi \bullet m_{b 1}$ is the rotation angle of the cradle, and $m_{b 1}$ is the rolling ratio of the pinion.

The tooth surface of the pinion is generated by the cutter head with a straight blade. In the $S_{p}$ coordinate system, the vector function, $\boldsymbol{r}_{p}$, of the cutting cone formed by the head cutter and its unit normal vector, $\mathbf{n}_{p}$, can be expressed as follows [7]:

$$
\begin{aligned}
& \mathbf{r}_{p}= {\left[\begin{array}{c}
\left(r_{c 1} \mp u_{p} \sin \alpha_{p}\right) \cos \theta_{p} \\
\left(r_{c 1} \mp u_{p} \sin \alpha_{p}\right) \sin \theta_{p} \\
-s_{p} \cos \alpha_{p} \\
1
\end{array}\right], } \\
& \mathbf{n}_{p}=\left[\begin{array}{c}
\cos \alpha_{p} \cos \theta_{p} \\
\cos \alpha_{p} \sin \theta_{p} \\
\mp \sin \alpha_{p}
\end{array}\right]
\end{aligned}
$$



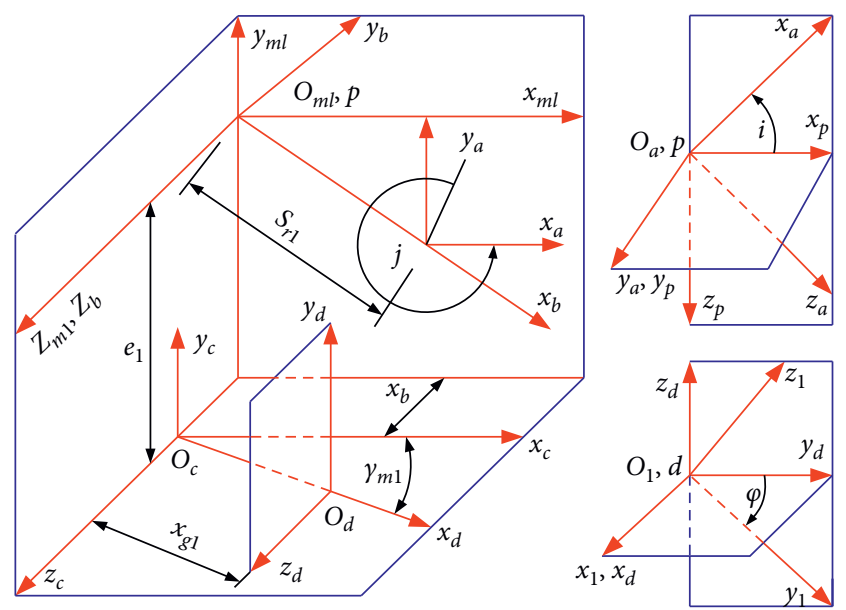

FIgURE 1: Coordinate systems applied for pinion generation.

where $s_{p}$ and $\theta_{p}$ are the Gaussian coordinates of the pinion tooth surface; $\alpha_{p}$ is the blade angle of the cutter head, and $r_{c 1}$ is the point radius of the cutter head.

The upper and lower signs in equation (1) correspond to the convex and concave surfaces of the pinion. They can form a mesh with the concave and convex surfaces of the gear, respectively. According to the coordinate systems applied for pinion generation shown in Figure 1, $\mathbf{r}_{p}$ and $\mathbf{n}_{p}$ are converted into the $S_{1}$ coordinate system. As a result, the tooth surface equation $\mathbf{r}_{1}$ and its unit normal vector $\mathbf{n}_{1}$ of the pinion in the $S_{1}$ coordinate system can be obtained as follows:
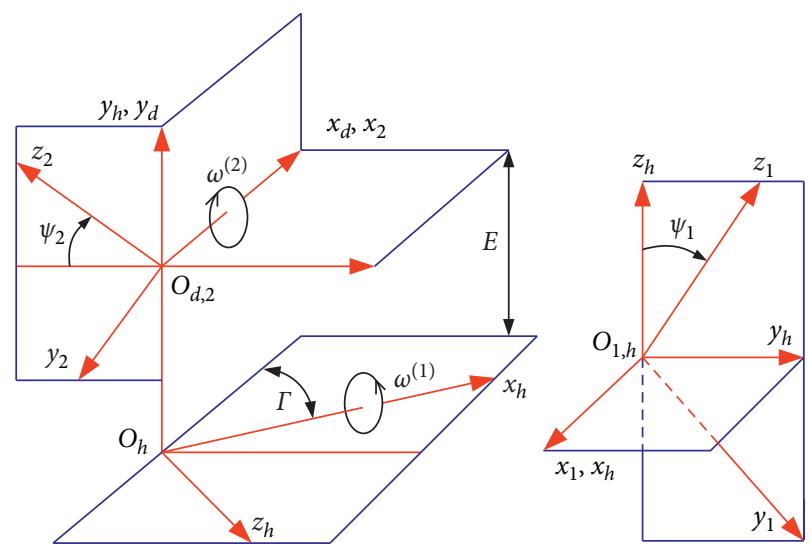

FIgURE 2: Coordinate system of gear pair meshing.

$$
\left\{\begin{array}{l}
\mathbf{r}_{1}\left(u_{p}, \theta_{p}, \varphi\right)=\mathbf{M}_{1 p} \mathbf{r}_{p}\left(u_{p}, \theta_{p}\right), \\
\mathbf{n}_{1}\left(u_{p}, \theta_{p}, \varphi\right)=\mathbf{L}_{1 p} \mathbf{n}_{p}\left(u_{p}, \theta_{p}\right), \\
f_{1}=\mathbf{n}_{1} \cdot\left(\frac{\partial \mathbf{r}_{1}(1: 3)}{\partial \varphi}\right),
\end{array}\right.
$$

where $f_{1}$ is the meshing equation when generating pinion [7], matrix $\mathbf{M}_{1 p}$ represents the transformation matrix from the coordinate system $S_{p}$ to $S_{1}$, and matrix $\mathbf{L}_{1 p}$ is the third-order submatrix of matrix $\mathbf{M}_{1 p} \cdot \mathbf{M}_{1 p}$ can be expressed as

$$
\begin{aligned}
\mathbf{M}_{1 p}= & {\left[\begin{array}{cccc}
1 & 0 & 0 & 0 \\
0 & \cos \varphi & -\sin \varphi & 0 \\
0 & \sin \varphi & \cos \varphi & 0 \\
0 & 0 & 0 & 0
\end{array}\right] \cdot\left[\begin{array}{cccc}
\cos \gamma_{m 1} & 0 & \sin \gamma_{m 1} & -x_{b} \sin \gamma_{m 1}-x_{g 1} \\
0 & 1 & 0 & e_{1} \\
-\sin \gamma_{m 1} & 0 & \cos \gamma_{m 1} & 0 \\
0 & 0 & 0 & -x_{b} \cos \gamma_{1}
\end{array}\right] } \\
& \cdot\left[\begin{array}{cccc}
\cos q & \sin q & 0 & 0 \\
-\sin q & \cos q & 0 & 0 \\
0 & 0 & 1 & 0 \\
0 & 0 & 0 & 1
\end{array}\right] \cdot\left[\begin{array}{cccc}
-\sin j & -\cos j & 0 & s_{r 1} \\
\cos j & -\sin j & 0 & 0 \\
0 & 0 & 1 & 0 \\
0 & 0 & 0 & 1
\end{array}\right] \cdot\left[\begin{array}{cccc}
\cos i & 0 & \sin i & 0 \\
0 & 1 & 0 & 0 \\
-\sin i & 0 & \cos i & 0 \\
0 & 0 & 0 & 1
\end{array}\right],
\end{aligned}
$$

where $q=q_{1}+f_{1} \bullet m_{b 1}$. In addition, when the pinion is generated by the duplex helical method, the cradle has an axial helical motion. Thus, $x_{b}$ can be expressed as

$$
x_{b}=x_{b 0}-h l \bullet \varphi \bullet m_{b 1} \text {. }
$$

\section{Solving the Contact Point of the Target Tooth Surface while considering the Transmission Error}

3.1. Solving the Contact Path. The coordinate system of gear pair meshing is shown in Figure 2, which establishes the tooth contact analysis (TCA). $S_{h}$ is a fixed coordinate system.
$S_{d}$ is an auxiliary coordinate system. $S_{1}$ is a moving coordinate system fixed to the pinion and rotates around the $x_{d^{-}}$ axis at an angular velocity $\omega^{(1)} . S_{2}$ is a moving coordinate system fixed to the gear and rotates around the $x_{h}$-axis at an angular velocity $\omega^{(2)}$. $\psi_{1}$ and $\psi_{2}$ are the rotation angles of the rotary motion of the pinion and gear, respectively.

According to the coordinate system of gear pair meshing shown in Figure 2, the tooth surface equation $\mathbf{r}_{1}$ and unit normal vector $\mathbf{n}_{1}$ of the pinion are converted into the meshing coordinate system $S_{h}$ to obtain $\mathbf{r}_{h 1}$ and $\mathbf{n}_{h 1}$ :

$$
\left\{\begin{array}{l}
\mathbf{r}_{h 1}\left(u_{p}, \theta_{p}, \varphi, \psi_{1}\right)=\mathbf{M}_{h 1} \mathbf{r}_{1}\left(u_{p}, \theta_{p}, \varphi\right), \\
\mathbf{n}_{h 1}\left(u_{p}, \theta_{p}, \varphi, \psi_{1}\right)=\mathbf{L}_{h 1} \mathbf{n}_{1}\left(u_{p}, \theta_{p}, \varphi\right),
\end{array}\right.
$$


where matrix $\mathbf{M}_{h 1}$ represents the transformation matrix from the $S_{1}$ to $S_{h}$ and matrix $\mathbf{L}_{h 1}$ is the third-order submatrix of matrix $\mathbf{M}_{h 1}$.

Similarly, the tooth surface equation $\mathbf{r}_{2}$ and its unit normal vector $\mathbf{n}_{2}$ of the gear are converted to the meshing coordinate system $S_{h}$ to get $\mathbf{r}_{h 2}$ and $\mathbf{n}_{h 2}$ :

$$
\left\{\begin{array}{l}
\mathbf{r}_{h 2}\left(u_{g}, \theta_{g}, \psi_{2}\right)=\mathbf{M}_{h 2} \mathbf{r}_{2}\left(u_{g}, \theta_{g}\right), \\
\mathbf{n}_{h 2}\left(u_{g}, \theta_{g}, \psi_{2}\right)=\mathbf{L}_{h 2} \mathbf{n}_{2}\left(u_{g}, \theta_{g}\right),
\end{array}\right.
$$

where matrix $\mathbf{M}_{h 2}$ is the transformation matrix from the $S_{2}$ to $S_{h}$ and matrix $\mathbf{L}_{h 1}$ is the third-order submatrix of matrix $\mathbf{M}_{h 1}$.

In the gear pair meshing process of a gear pair, the two tooth surfaces should have the same radial vector and unit normal vector at any meshing point; hence, the TCA equation can be constructed [22]:

$$
\left\{\begin{array}{l}
\mathbf{r}_{h 1}\left(u_{p}, \theta_{p}, \varphi, \psi_{1}\right)=\mathbf{r}_{h 2}\left(u_{p}, \theta_{p}, \psi_{2}\right), \\
\mathbf{n}_{h 1}\left(u_{p}, \theta_{p}, \varphi, \psi_{1}\right)=\mathbf{n}_{h 2}\left(u_{p}, \theta_{p}, \psi_{2}\right) .
\end{array}\right.
$$

The transmission error curve and contact path can be obtained by solving equation (7). The tooth surfaces of the pinion and gear obtained by the initial machine tool parameters are defined as the initial pinion tooth surface $\Sigma^{(0)}$ and initial gear tooth surface, respectively. The radial vector $\mathbf{r}_{t}^{(0)}$ and unit normal vector $\mathbf{n}_{t}^{(0)}$ of the discrete point of the contact path on the initial pinion tooth surface $\Sigma^{(0)}$ can be obtained by the TCA. Here, $t$ represents the number of discrete points on the contact path, and $t=1,2, \ldots, m$. The radial vectors $\mathbf{r}_{t}^{(0)}$ of the discrete points of the contact path are converted into the coordinate system of the rotating projection surface using equation (8). Consequently, the contact points $\left(L_{t}, R_{t}\right)$, which correspond to $\mathbf{r}_{t}^{(0)}$, are obtained as follows:

$$
\left\{\begin{array}{l}
L_{t}=\mathbf{r}_{t}^{(0)}(1), \\
R_{t}=\sqrt{\left(\mathbf{r}_{t}^{(0)}(2)\right)^{2}+\left(\mathbf{r}_{t}^{(0)}(3)\right)^{2}} .
\end{array}\right.
$$

3.2. Solution of the Contact Point of the Target Tooth Surface Driven by the Transmission Error. The transmission error $\triangle \psi_{2}\left(\psi_{1}\right)$ of the gear pair is defined as the difference between the actual rotation angle $\psi_{2}$ and theoretical rotation angle $\left(\mathrm{z}_{1} / \mathrm{z}_{2}\right) \bullet \psi_{1}$ of the gear when the pinion rotates through an angle $\psi_{1}[23]$ :

$$
\Delta \psi_{2}\left(\psi_{1}\right)=\left(\psi_{2}-\psi_{2}^{(0)}\right)-\left(\frac{z_{1}}{z_{2}}\right) \bullet\left(\psi_{1}-\psi_{1}^{(0)}\right)
$$

where $\psi_{1}^{(0)}$ is the initial rotation angle of the pinion, $\psi_{2}^{(0)}$ is the initial rotation angle of the gear, $Z_{1}$ is the number of pinions, and $Z_{2}$ is the number of gears.

As shown in Figure 3, the second-order parabola transmission error curve is predicted on the concave and convex surface of the initial pinion to obtain the target pinion tooth surface. The contact point of the target pinion

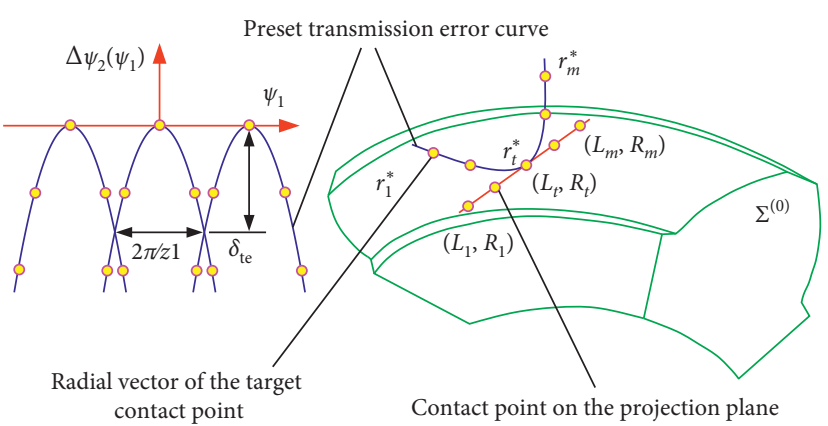

FIgURE 3: Preset second-order parabola transmission error curve.

tooth surface will coincide with the contact point of the initial pinion $\left(L_{t}, R_{t}\right)$. Given the coordinate value $\left(\delta_{\mathrm{te}}, \pi / z_{1}\right)$ of the meshing transition point of the parabolic transmission error curve, the second-order parabolic transmission error curve can be expressed as

$$
\Delta \psi_{2}\left(\psi_{1}\right)=-\delta_{\mathrm{te}} \cdot\left(\frac{z_{1}^{2}}{\pi^{2}}\right) \cdot\left(\psi_{1}-\psi_{1}^{0}\right)^{2},
$$

where $\delta_{\mathrm{te}}$ is the amplitude of the meshing transition point of the transmission error curve. Combining equation (9) and equation (10), the motion relationship between the gear and the pinion can be obtained:

$$
\psi_{2}=\psi_{2}^{(0)}+\left(\frac{z_{1}}{z_{2}}\right) \bullet\left(\psi_{1}-\psi_{1}^{(0)}\right)-\delta_{t e} \bullet\left(\frac{z_{1}^{2}}{\pi^{2}}\right) \bullet\left(\psi_{1}-\psi_{1}^{0}\right)^{2} .
$$

Combining equation (8) with equation (11), the tooth surface equation $\mathbf{r}_{2}$ of the initial gear can be converted into the pinion coordinate system $S_{1}$ according to the meshing coordinate system as shown in Figure 2. Consequently, the radial vector $\mathbf{r}_{t}^{*}$ and unit normal vector $\mathbf{n}_{t}^{*}$ of the target pinion tooth surface $\Sigma^{*}$ corresponding to the contact point $\left(L_{t}, R_{t}\right)$ of the initial pinion tooth surface point can be obtained can be obtained using the following equations:

$$
\left\{\begin{array}{l}
\mathbf{r}_{t}^{*}=\mathbf{M}_{12} \mathbf{r}_{2}\left(u_{g}, \theta_{g}\right), \\
\mathbf{n}_{t}^{*}=\mathbf{L}_{12} \mathbf{n}_{2}\left(u_{g}, \theta_{g}\right) \\
f_{t}^{*}\left(u_{g}, \theta_{g}, \psi_{2}\right)=\mathbf{n}_{t}^{*} \cdot\left(\frac{\partial \mathbf{r}_{t}^{*}(1: 3)}{\partial \psi_{2}}\right), \\
\psi_{2}=\psi_{2}^{(0)}+\left(\frac{z_{1}}{z_{2}}\right)\left(\psi_{1}-\psi_{1}^{(0)}\right)-\delta_{t e}\left(\psi_{1}-\psi_{1}^{0}\right)^{2}, \\
\left(\mathbf{r}_{t}^{*}\right)_{x}=L_{t}, \\
\sqrt{\left(\mathbf{r}_{t}^{*}\right)_{y}^{2}+\left(\mathbf{r}_{t}^{*}\right)_{z}^{2}}=R_{t},
\end{array}\right.
$$

where $f_{1}^{*}$ is the meshing equation in the coordinate system of gear pair meshing [24]. Matrix $\mathbf{M}_{12}$ represents the 
transformation matrix from $S_{2}$ to $S_{1}$. Meanwhile, matrix $\mathbf{L}_{12}$ is the third-order submatrix of matrix $\mathbf{M}_{12}$. Matrix $\mathbf{M}_{12}$ can be expressed as

$$
\mathbf{M}_{12}=\left[\begin{array}{cccc}
1 & 0 & 0 & 0 \\
0 & \cos \psi_{1} & -\sin \psi_{1} & 0 \\
0 & \sin \psi_{1} & \cos \psi_{1} & 0 \\
0 & 0 & 0 & 0
\end{array}\right] \bullet\left[\begin{array}{cccc}
\cos \Gamma & 0 & \sin \Gamma & 0 \\
0 & 1 & 0 & E \\
-\sin \Gamma & 0 & \cos \Gamma & 0 \\
0 & 0 & 0 & 0
\end{array}\right] \bullet\left[\begin{array}{cccc}
1 & 0 & 0 & 0 \\
0 & -\cos \psi_{2} & \sin \psi_{2} & 0 \\
0 & -\sin \psi_{2} & -\cos \psi_{2} & 0 \\
0 & 0 & 0 & 1
\end{array}\right] .
$$

\section{Nonlinear Accurate Optimization of Transmission Error}

The flow chart of the nonlinear optimization method for the transmission error of hypoid gears machined by the duplex helical method is shown in Figure 4.

4.1. Optimization Model. Ease-off is defined as the deviation of the corresponding contact point between the target and the initial tooth surfaces [25]. In the past, the optimization of the transmission error of the spiral bevel gear cut by the fivecut method has considered the deviation of the discrete points of the entire tooth surface $[6,9,20]$. For the simultaneous machining of concave and convex surfaces, the duplex helical method is applied. A method of correcting only the ease-off of the contact point between the target pinion tooth surface $\Sigma^{*}$ and initial pinion tooth surface $\Sigma^{(0)}$ is proposed. The proposed method is more practical and effective than correcting the deviation of the discrete points of the entire tooth surface. By correcting the machine parameters, the ease-off of the contact point can be minimized to optimize the transmission error. The radial vector $\mathbf{r}_{t}^{(0)}$ and its unit normal vector $\mathbf{n}_{t}^{(0)}$ of the contact point on the initial pinion tooth surface are expressed as

$$
\left\{\begin{array}{c}
\mathbf{r}_{t}^{(0)}=\mathbf{r}_{t}^{(0)}\left(\varepsilon_{t}, \mathbf{x}\right) \\
\mathbf{n}_{t}^{(0)}=\mathbf{n}_{t}^{(0)}\left(\varepsilon_{t}, \mathbf{x}\right)
\end{array}\right\}, \quad(t=1,2, \ldots, m)
$$

where $e_{t}=\left(u_{p}, \theta_{p}, \varphi\right)$ and $\boldsymbol{x}=\left[q_{1}, s_{r 1}, m_{b 1}, \gamma_{m 1}, x_{b 1}, x_{g 1}, e_{1}, h l\right.$, $i, j]$, which is a vector containing 10 machine tool parameters. Meanwhile, $t(t=1,2, \ldots, m)$ can be expressed as the $t$ th contact point.

The deviation correction model for contact points is shown in Figure 5. $\mathbf{r}_{t}^{(0)}$ and $\mathbf{r}_{t}^{*}$ represent the position vectors of the contact point on the initial pinion tooth surface $\Sigma^{(0)}$ and target pinion tooth surface $\Sigma^{*}$, respectively. $\mathbf{h}_{t}$ is the deviation vector of the corresponding contact point. Thus, the position vector $\mathbf{r}_{t}^{*}$ of the contact point of the target pinion tooth surface $\Sigma^{*}$ can be expressed as

$$
\mathbf{r}_{t}^{*}=\mathbf{r}_{t}^{(0)}\left(\varepsilon_{t}, \mathbf{x}\right)+\mathbf{n}_{t}^{(0)}\left(\varepsilon_{t}, \mathbf{x}\right) \mathbf{h}_{t} .
$$

The Gaussian coordinate parameters $u_{p}$ and $\theta_{p}$ of the initial tooth surface are located along the tangential plane of the tooth surface. Therefore, parameter $\varepsilon_{t}\left(u_{p}, \theta_{p}, \varphi\right)$ can be eliminated as follows:

$$
\left\{\begin{array}{l}
{\left[\mathbf{r}_{t}^{*}-\mathbf{r}_{t}^{(0)}\left(\varepsilon_{t}, \mathbf{x}\right)\right] \cdot\left(\frac{\partial \mathbf{r}_{t}^{(0)}\left(\varepsilon_{t}, \mathbf{x}\right)}{\partial u_{p}}\right)=0,} \\
{\left[\mathbf{r}_{t}^{*}-\mathbf{r}_{t}^{(0)}\left(\varepsilon_{t}, \mathbf{x}\right)\right] \bullet\left(\frac{\partial \mathbf{r}_{t}^{(0)}\left(\varepsilon_{t}, \mathbf{x}\right)}{\partial \theta_{p}}\right)=0,} \\
f\left(\varepsilon_{t}, \mathbf{x}\right)=0,
\end{array}\right.
$$

where $f\left(\varepsilon_{t}, \mathbf{x}\right)$ is the meshing equation for cutting the initial pinion tooth surface.

Therefore, the deviation vector, $\mathbf{h}_{t}$, of the tooth surface can be expressed as

$$
\mathbf{h}_{t}=\left[\mathbf{r}_{t}^{*}-\mathbf{r}_{t}^{(0)}\left(\varepsilon_{t}(\mathbf{x}), \mathbf{x}\right)\right] \mathbf{n}_{t}^{(0)}\left(\varepsilon_{t}(\mathbf{x}), \mathbf{x}\right) .
$$

Let $\mathbf{h}(\mathbf{x})=\mathbf{h}_{t}(\mathbf{x})$ be the nonlinear least squares optimization model for the synchronous optimization of the transmission errors. The concave and convex surfaces of the pinion are established by taking the machine tool parameters as design variables and the minimum sum of the residual squares of $\mathbf{h}(\mathbf{x})$ as the objective function:

$$
\mathbf{x}^{*}=\arg \min f(\mathbf{x}) \text { with } f(\mathbf{x})=\frac{1}{2} \mathbf{h}(\mathbf{x})^{T} \mathbf{h}(\mathbf{x}) .
$$

4.2. Selection of Machine Tool Parameters. The machining parameters of the generated tooth surface include the headcutter and machine tool parameters. The cutter head parameters include the tooth profile angle $\alpha_{p}$ and point radius $r_{c 1}$. When $\alpha_{p}$ and $r_{c 1}$ are used as optimization variables, the geometry of the cutter head needs to be changed, that is, the cutting edge of the cutter head must be ground. However, grinding the cutting edge is time consuming, expensive, and difficult. Therefore, $\alpha_{p}$ and $r_{c 1}$ are not used as optimization variables. Instead, optimization variables are only selected from machine tool parameters. In addition, when selecting optimization variables, the coupling between machine tool parameters is considered; machine tool parameters that strongly influence the normal deviation of the tooth surface are preferred. If the optimization result does not satisfy the requirements, the optimization variables are sequentially increased according to the influence degree of the machine tool parameters on the normal deviation of the tooth surface. The degree of influence of the machine tool parameters on the normal deviation of the tooth surface is defined by the 


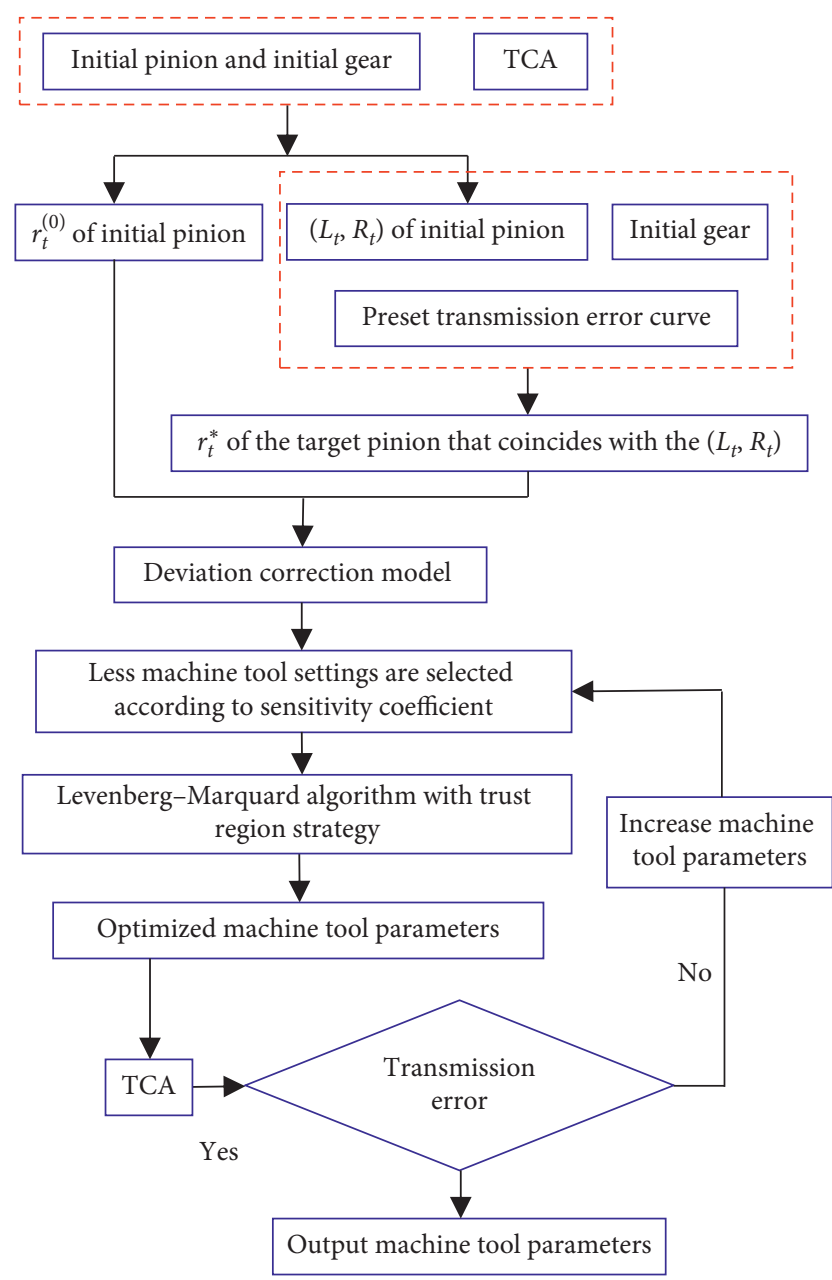

FIGURE 4: Flow chart of the nonlinear optimization method for the transmission error of hypoid gears machined by the duplex helical method.

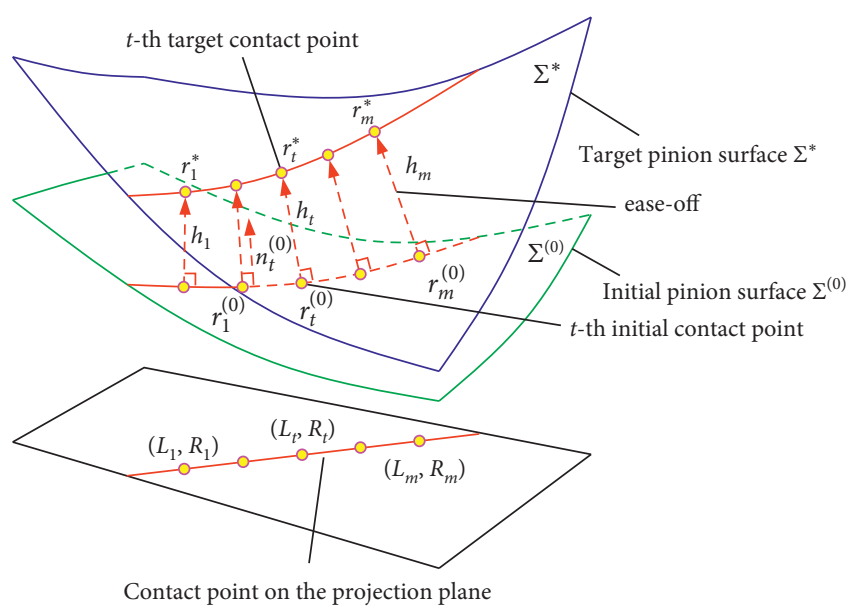

FIGURE 5: Deviation correction model for contact points.

sensitivity coefficient. Assume that the machine tool parameter change is $c p$ and the sum of the absolute values of the normal deviations that cause discrete points on the tooth surface is $s d$; then, the sensitivity coefficient is $c p / s d$. We set the angle parameter change amount to $0.05^{\circ}$, the linear parameter error to $0.05 \mathrm{~mm}$, and the roll ratio error to 0.005 . Then, the sensitivity coefficients of the machine tool parameters to the tooth surface normal deviation are listed as in Table 1.

4.3. Optimization Algorithm. Presently, the nonlinear least squares problem is typically solved using an iterative algorithm. However, when an iterative algorithm is used to solve equation (18), the solution of the equation set is unstable due to the ill-conditions of the coefficient matrix. Thus, it is difficult to solve the accurate and stable corrections of the machine tool parameters [17-19]. To ensure the accuracy of the solution, the Levenberg-Marquard algorithm with a trust region strategy is used to solve equation (18) to ensure the positive definiteness of the Hessian matrix at each step in the iterative process. This approach ensures an accurate and efficient iteration for each step. Using the quadratic approximation function $q_{k}(\mathbf{x})$, the solution of the objective function $f(\mathbf{x})$ of equation (18) can be transformed into a solution to the subproblem for the following trust region:

$$
\begin{gathered}
\mathbf{s}_{k}=\underset{\arg \min }{ } q_{k}(\mathbf{s})=f\left(\mathbf{x}_{k}\right)+\mathbf{g}_{k} \mathbf{s}+\frac{1}{2} \mathbf{s}^{T} \mathbf{G}_{k} \mathbf{s}, \\
\text { s.t. }\|\mathbf{s}\| \leq \Delta k,
\end{gathered}
$$

where $k$ is the number of iterations and $f_{k}=f\left(\mathbf{x}_{k}\right)$. Furthermore, $\mathbf{s}=\mathbf{x}-\mathbf{x}_{k}$, which is the iterative step and is the variable to be determined. $\mathbf{g}_{k}$ and $\mathbf{G}_{\mathrm{k}}$ are the Jacobian and Hessian matrices of the objective function $f_{k}(\mathbf{x})$ at $\mathbf{x}_{k}$, respectively. $\mathbf{h}\left(\mathbf{x}_{\mathrm{k}}\right)$ is the ease-off vector of the tooth surface. Additionally, $\mathbf{g}_{k}=\mathbf{h}\left(\mathbf{x}_{k}\right)^{\mathrm{T}} \mathbf{J}\left(\mathbf{x}_{k}\right)$ and $\mathbf{G}_{\mathrm{k}}=\mathbf{J}\left(\mathbf{x}_{k}\right)^{\mathrm{T}} \mathbf{J}\left(\mathbf{x}_{k}\right) . \Delta k$ is the radius of the trust region. When $\Delta \mathrm{k}$ changes, the solution of equation (19) is a spatial curve, which is the optimal curve shown in Figure 6. To ensure the positive characterization of $\mathbf{G}_{k}$, the solution of equation (19) is equivalent to solving the following equation:

$$
\mathbf{s}_{k}=\mathbf{s}\left(\mu_{k}\right)=-\left(\mathbf{G}_{k}+\mu_{k} \mathbf{I}\right)^{-1} \mathbf{g}_{k}^{T},
$$

where $\mu_{k} \geq 0$ is the damping coefficient equivalent to the positive parameter. The damping coefficient is used to ensure the regularity of $\boldsymbol{G}_{k}$ in each iteration and $\boldsymbol{I}$ is the identity matrix. According to equation (20), $\mathrm{s}_{k}^{G N}=-\mathbf{G}_{k}^{-1} \mathbf{g}_{k}^{T}$ is the Gauss-Newton iteration step when $\mu_{k}=0$. $\mathbf{s}_{k}^{C P}=-\left(1 / \mu_{k}\right) \mathbf{g}_{k}^{T}$ is a small step in the direction of steepest descent when $\mu_{k}$ is large. Therefore, the Levenberg-Marquard algorithm with a trust region strategy focuses on two strategies. The first strategy involves finding the iterative step size, $\mathbf{s}\left(u_{k}\right)$, for the given trust region radius $\triangle k$, which is the solution of equation (20). The second strategy involves updating $\triangle k$.

To solve equation (20), the single dogleg method [26] and double dogleg method [27] are used. Figure 6 shows the tangent single dogleg method. When solving equation (20) by the tangent single dogleg method, the optimal curve $s$ $(\triangle k)$ is replaced with the polyline $s_{y}(\triangle k)$. The polyline $s_{y}$ $(\triangle k)$ can be obtained through the following steps. First, the 
TABle 1: Sensitivity coefficient of machine tool parameters to normal deviation of tooth surface.

\begin{tabular}{lc}
\hline Machine tool parameters & $\begin{array}{c}\text { Sensitivity } \\
\text { coefficient }\end{array}$ \\
\hline Tilt angle $i\left(^{\circ}\right)$ & 271 \\
Swivel angle $j\left(^{\circ}\right)$ & 102 \\
Radial distance $s_{r 1}(\mathrm{~mm})$ & 9 \\
Center roll position $q_{1}\left(^{\circ}\right)$ & 1.2 \\
Work offset $e_{1}(\mathrm{~mm})$ & 4.9 \\
Machine center to back $x_{g 1}(\mathrm{~mm})$ & 2.3 \\
Sliding base $x_{b 1}(\mathrm{~mm})$ & 1.7 \\
Ratio of roll $m_{b 1}$ & 116 \\
Machine root angle $\gamma_{m 1}\left({ }^{\circ}\right)$ & 291 \\
Velocity coefficient of helical motion $h l$ & 2.4 \\
$(\mathrm{~mm} /$ rad) & \\
\hline
\end{tabular}

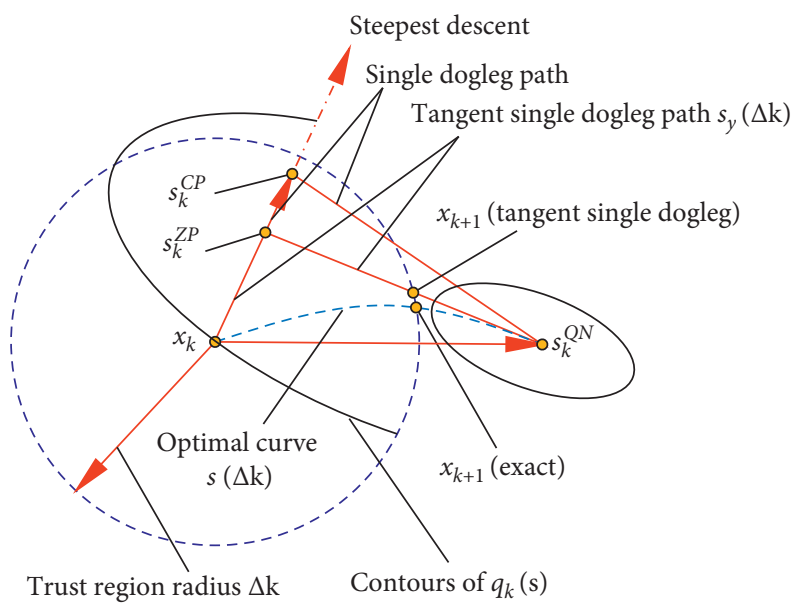

Figure 6: Tangent single dogleg method.

Gauss-Newton point s GN in the Gauss-Newton direction is taken as the starting point to form the tangent line of the optimal curve $s(\Delta \mathrm{k})$. Second, the tangent line is projected onto the plane spanned by the fastest descending direction $-\mathbf{g}_{k}^{T}$ and the Gauss-Newton direction $-\mathbf{G}_{k}^{-1} \mathbf{g}_{k}^{T}$; the projected line has an intersection point $\mathbf{s}_{k}^{Z P}$ with $-\mathbf{g}_{k}^{T}$. Finally, $s_{y}(\Delta k)$ can be obtained by connecting the initial point $\mathbf{x}_{k}, \mathbf{s}_{k}^{Z P}$ and $\mathbf{s}_{k}^{G N}$. Compared with the single dogleg method, the tangent single dogleg method yields $s_{y}(\triangle k)$ closer to the Gauss-Newton direction, in other words, closer to the optimal curve. Thus, the tangent single dogleg method is better with convergence.

When the Gauss-Newton step $\mathbf{s}_{k}^{\mathrm{QN}}$ is within the trust region radius $\Delta k$, that is, if $\left\|\mathbf{s}_{k}^{Q N}\right\| \leq \Delta k$, then the iterative step is the optimal solution of equation (20), which is $\mathbf{s}_{k}^{\mathrm{QN}}$. When $\left\|\mathbf{s}_{k}^{\mathrm{QN}}\right\|>\Delta k$, the tangent single dogleg method can be used to determine the approximate iteration step size. The tangent simple dogleg path sy $(\triangle \mathrm{k})$ consists of two line segments, which replaces the optimal curve $\mathbf{s}(\triangle k)$, as shown in Figure 6. Equation (20) is the parametric equation of the optimal curve; by applying $u_{k}$, this makes $\|\boldsymbol{s}\|=\Delta k$. The tangent of the curve at any point is

$$
\mathbf{s}_{k}^{\prime}=\left(\mathbf{G}_{k}+\mu_{k} \mathbf{I}\right)^{-1}\left(\mathbf{G}_{k}+\mu_{k} \mathbf{I}\right)^{-1} \mathbf{g}_{k}^{T}, \quad \mu \geq 0,
$$

and when considering $\mu_{k}=0$, the tangent of the point $\mathbf{s}_{k}^{\mathrm{QN}}$ is

$$
\left.\mathbf{s}_{k}^{\prime}\right|_{\mu=0}=\mathbf{G}_{k}^{-1} \mathbf{G}_{k}^{-1} \mathbf{g}_{k}^{T} .
$$

Then, the equation represented by line $\mathbf{s}_{k}^{Z P} \mathbf{s}_{k}^{\mathrm{QN}}$ is

$$
y=\mathbf{G}_{k}^{-1} \mathbf{g}_{k}^{T}+\alpha \mathbf{G}_{k}^{-1} \mathbf{G}_{k}^{-1} \mathbf{g}_{k}^{T}, \quad \alpha \in R
$$

The line is projected onto the plane formed by $\left[-\mathbf{g}_{k}^{T}, \mathbf{G}_{k}^{-1} \mathbf{g}_{k}^{T}\right]$, and let $A=\left[-\mathbf{g}_{k}^{T},-\mathbf{G}_{k}^{-1} \mathbf{g}_{k}^{T}\right]$. Then, $P=A$ $\left(A^{T} A\right)^{-1} A^{T}$ is the projection matrix of the $\mathrm{z}$-dimensional vector onto the A plane. If the steepest descending direction $-\mathbf{g}_{k}^{T}$ is not parallel to the Gaussian iteration direction $-\mathbf{G}_{k}^{-1} \mathbf{g}_{k}^{T}$, then $P\left(-\mathbf{G}_{k}^{-1} \mathbf{g}_{k}^{T}+\alpha \mathbf{G}_{k}^{-1} \mathbf{G}_{k}^{-1} \mathbf{g}_{k}^{T}\right)$ must have an intersection with $-\mathbf{g}_{k}^{T}$. Thus, the solution of the following equation must be unique:

$$
\beta\left(-\mathbf{g}_{k}^{T}\right)=\mathbf{G}_{k}^{-1} \mathbf{g}_{k}^{T}+\alpha P \mathbf{G}_{k}^{-1} \mathbf{G}_{k}^{-1} \mathbf{g}_{k}^{T} .
$$

Let $\mathbf{d}=\mathbf{G}_{k}^{-1} \mathbf{g}_{k}^{T}$ and $\mathbf{e}=\mathbf{P G}_{k}^{-1} \mathbf{G}_{k}^{-1} \mathbf{g}_{k}^{T}$. Then, equation (24) can be transformed into

$$
-\beta \mathbf{g}_{k}^{T}=-\mathbf{d}+\alpha \mathbf{e} .
$$

If $P<z$, then the first and $p$-th components of vector $\mathbf{g}_{k}^{T}$, $\mathbf{d}$ and $\mathbf{e}$, respectively, are used to establish the system of equations:

$$
\left\{\begin{array}{l}
-\beta \mathbf{g}_{k}^{T(1)}=-\mathbf{d}^{(1)}+\alpha \mathbf{e}^{(1)}, \\
-\beta \mathbf{g}_{k}^{T(p)}=-\mathbf{d}^{(p)}+\alpha \mathbf{e}^{(p)} .
\end{array}\right.
$$

If $\mathbf{g}_{k}^{T(1)} \mathbf{e}^{(p)}-\mathbf{g}_{k}^{T(p)} \mathbf{e}^{(1)} \neq 0$, then equation (26) has a solution:

$$
\beta=\frac{\mathbf{d}^{(p)} \mathbf{e}^{(1)}-\mathbf{d}^{(1)} \mathbf{e}^{(p)}}{\mathbf{g}_{k}^{T(1)} \mathbf{e}^{(p)}-\mathbf{g}_{k}^{T(p)} \mathbf{e}^{(1)}} .
$$

Then, point $\mathbf{s}_{k}^{Z P}=-\beta \mathbf{g}_{k}^{T}$ can be determined. Therefore, the solution $s_{k}$ of equation (20) is the iteration step where the trust region can be expressed as follows:

$$
\mathbf{s}_{k}= \begin{cases}\frac{\Delta k}{\left\|\mathbf{s}_{k}^{Z P}\right\|} \mathbf{s}_{k}^{Z P}, & \Delta k \leq\left\|\mathbf{s}_{k}^{Z P}\right\|, \\ \mathbf{s}_{k}^{\mathrm{QN}}, & \Delta k \geq\left\|\mathbf{s}_{k}^{\mathrm{QN}}\right\|, \\ \mathbf{s}_{k}^{Z P}+\tau\left(\mathbf{s}_{k}^{\mathrm{QN}}-\mathbf{s}_{k}^{Z D}\right), & \left\|\mathbf{s}_{k}^{Z P}\right\| \leq \Delta k \leq\left\|\mathbf{s}_{k}^{\mathrm{QN}}\right\|, \\ \tau \text { made }\left\|\mathbf{s}_{k}\right\|=\Delta k . & \end{cases}
$$

The second problem involves updating the trust region radius $\triangle k$, after controlling the iterative step by the tangent single dogleg method. In other words, we update the damping parameter $\mu_{k}$. When the trust region algorithm is used, the damping parameter $\mu_{k}$, can be updated by the increment $\rho_{k}$ of the ratio of the objective function $f(\mathbf{x})$ and the quadratic approximation function $q_{k}(\mathbf{x})$ : 


$$
\rho_{k}=\frac{\Delta f(\mathbf{x})}{\Delta q_{k}(\mathbf{x})}=\frac{f\left(\mathbf{x}_{k}+\mathbf{s}_{k}\right)-f\left(\mathbf{x}_{k}\right)}{q_{k}\left(\mathbf{s}_{k}\right)-q_{k}(0)} .
$$

Each iterative process of the Levenberg-Marquard algorithm with trust region strategy is achieved through the following steps. First, $\mu_{k}$ is given an initial value. Second, $\boldsymbol{s}_{k}$ can be calculated according to the value of the previous iteration step. Afterwards, $\mu_{k}$ is adjusted according to the value of $\rho_{k}$. Finally, $s_{k}$ is calculated according to the adjusted $\mu_{k}$. The update rules of parameter $\mu_{k}$ are as follows [28]:

$$
\mu_{k+1}= \begin{cases}0.1 \mu_{k}, & \rho_{k}>0.75 \\ \mu_{k}, & 0.25 \leq \rho_{k} \leq 0.75 \\ 10 \mu_{k}, & \rho_{k}<0.25\end{cases}
$$

\section{Numerical Example}

5.1. Nonlinear Optimization of Transmission Error. In this study, we used a pair of hypoid gear pairs machined by the forming method for the gear and the duplex helical method for the pinion as an example. Table 2 lists the main geometric parameters of the hypoid gear pair; Table 3 lists the parameters of the preset second-order parabolic transmission error curve; and Table 4 is the initial head-cutter parameters and machine tool parameters of the hypoid gear pair.

The target pinion can be obtained using the two constraints of the preset transmission error curve and the coincidence of the contact line with the initial pinion. According to the flowchart shown in Figure 4, the Levenberg-Marquard algorithm with trust region strategy is used to optimize the transmission error and converges after 35 iterations. The deviation of the concave surface is reduced from a maximum of -13 um to a maximum of $-1.3 \mathrm{um}$; and the deviation of the convex surface is reduced from a maximum of $12.9 \mu \mathrm{m}$ to a maximum of $1.5 \mu \mathrm{m}$. In addition, the sequential quadratic programming algorithm $[17,18]$ was also used to optimize the transmission error; and the results were compared with those obtained using the Levenberg-Marquardt algorithm with the trust region strategy. After optimization using the sequential quadratic programming algorithm, the deviation of the concave surface is reduced from a maximum of $-13 \mu \mathrm{m}$ to a maximum of $-5.6 \mu \mathrm{m}$; and the deviation of the convex surface is reduced from a maximum of $12.9 \mathrm{um}$ to a maximum of $4.5 \mathrm{um}$. The comparison between the Levenberg-Marquard algorithm with trust region strategy and the sequential quadratic programming algorithm shows that the Levenberg-Marquard algorithm with trust region strategy has a better optimization effect. The optimized machine tool parameters of the pinion are listed in Table 5. TCA was applied to the hypoid gear pair that corresponding to the initial and optimized machine tool parameters. The optimized transmission error curve obtained by the Levenberg-Marquardt algorithm with trust region strategy was defined as optimized transmission error curve 1 , whereas that obtained by the sequential quadratic programming algorithm was defined as optimized transmission error curve 2 . The transmission error curve obtained from the initial machine tool parameters was defined as the initial transmission error curve. The

\begin{tabular}{|c|c|c|}
\hline & Gear & Pinion \\
\hline Number of teeth & 43 & 8 \\
\hline Module & \multicolumn{2}{|c|}{6.861} \\
\hline Face width (mm) & 41 & 44.691 \\
\hline Mean cone distance $(\mathrm{mm})$ & 130.762 & 128.323 \\
\hline Pinion offset $(\mathrm{mm})$ & \multicolumn{2}{|c|}{25.4} \\
\hline Mean spiral angle $\left({ }^{\circ}\right)$ & 33.8 & 45 \\
\hline Hand of spiral & Right & Left \\
\hline Hand of spiral $\left({ }^{\circ}\right)$ & \multicolumn{2}{|c|}{90} \\
\hline Pitch angle $\left({ }^{\circ}\right)$ & 77.215 & 12.549 \\
\hline Face angle of blank $\left({ }^{\circ}\right)$ & 78.124 & 16.154 \\
\hline Root angle $\left({ }^{\circ}\right)$ & 73.547 & 11.655 \\
\hline
\end{tabular}

TABLE 2: Main geometric parameters of the hypoid gear pairs.

TABle 3: Parameters of the preset second-order parabolic transmission error curve.

\begin{tabular}{lcc}
\hline & Concave & Convex \\
\hline Amplitude of meshing transition point $\left({ }^{\prime \prime}\right)$ & 5 & 6 \\
\hline
\end{tabular}

transmission error curve obtained from the initial machine tool parameters is was defined as the initial transmission error curve. Figure 7 shows the initial and preset curves, as well as optimized transmission error curves 1 and 2, for the concave surfaces of the pinion. Figure 8 shows the initial and preset curves, as well as optimized transmission error curves 1 and 2, for the convex surfaces of the pinion. Lines $\bigcirc, \boldsymbol{0}, \square$, and $\Delta$ in Figures 7 and 8 correspond to the initial curve, preset curve, optimized transmission error curve 1 , and optimized transmission error curve 2, respectively.

Furthermore, Figure 7, Figure 8, and Table 3 show that the Levenberg-Marquard algorithm with trust region strategy is better than sequential quadratic programming algorithm in optimizing transmission error. Therefore, the following content only discusses the optimization effect of the Levenberg-Marquard algorithm on the transmission error. Figures 7 and 8 show that the optimized transmission error curve 1 is consistent with the preset transmission error curve. Further analysis of the transmission error curve was conducted, and the results are summarized in Table 6. When comparing with the preset values, it was found that the amplitudes of the meshing transition points of the concave and convex surfaces of the optimized pinion differ by $0.1^{\prime \prime}$ and $0.2^{\prime \prime}$, i.e., $2 \%$ and $3.3 \%$, respectively. The amplitudes of the maximum transmission error of the concave and convex surfaces of the optimized pinion differ by $0.1^{\prime \prime}$ and $0.5^{\prime \prime}$, i.e., $1.2 \%$ and $4.6 \%$, respectively. When comparing with the initial values, the maximum amplitude and the amplitude of the meshing transformation point of optimized transmission error curve 1 are reduced. When performing further analysis of the transmission errors in Table 6, they were compared with the initial transmission error curve. The amplitudes of the meshing transition points of the concave and convex surfaces of the optimized pinion are reduced by $7.2^{\prime \prime}$ and $4.5^{\prime \prime}$, i.e., $59.5 \%$ and $43.7 \%$, respectively. The amplitudes of the maximum transmission error for the concave and convex surfaces of the optimized pinion are reduced by $11.3^{\prime \prime}$ 
TABLE 4: Initial head-cutter and machine tool parameters of the hypoid gear pair.

\begin{tabular}{|c|c|c|c|c|}
\hline \multirow[b]{2}{*}{ Blade angle $a_{p}\left({ }^{\circ}\right)$} & \multicolumn{2}{|c|}{ Gear } & \multicolumn{2}{|c|}{ Pinion } \\
\hline & $\begin{array}{c}\text { Concave } \\
20\end{array}$ & $\begin{array}{c}\text { Convex } \\
25\end{array}$ & $\begin{array}{c}\text { Concave } \\
20\end{array}$ & $\begin{array}{c}\text { Convex } \\
25\end{array}$ \\
\hline Point radius $r_{c 1}(\mathrm{~mm})$ & 115.824 & 112.776 & 114.966 & 112.206 \\
\hline Tilt angle $i\left(^{\circ}\right)$ & \multicolumn{2}{|r|}{ 然 } & \multicolumn{2}{|c|}{15.572} \\
\hline Swivel angle $j\left({ }^{\circ}\right)$ & \multicolumn{2}{|c|}{0} & \multicolumn{2}{|c|}{-35.332} \\
\hline Radial distance $s_{r 1}(\mathrm{~mm})$ & \multicolumn{2}{|c|}{116.573} & \multicolumn{2}{|c|}{117.385} \\
\hline Center roll position $q_{1}\left(^{\circ}\right)$ & \multicolumn{2}{|c|}{60.465} & \multicolumn{2}{|c|}{65.626} \\
\hline Work offset $e_{1}(\mathrm{~mm})$ & \multicolumn{2}{|c|}{0} & \multicolumn{2}{|c|}{28.568} \\
\hline Machine center to back $x_{q 1}(\mathrm{~mm})$ & \multicolumn{2}{|c|}{3.1416} & \multicolumn{2}{|c|}{-0.147} \\
\hline Sliding base $x_{b 1}(\mathrm{~mm})$ & \multicolumn{2}{|c|}{0} & \multicolumn{2}{|c|}{16.637} \\
\hline Ratio of roll $m_{b 1}$ & \multicolumn{2}{|c|}{0} & \multicolumn{2}{|c|}{5.326} \\
\hline Machine root angle $\gamma_{m 1}\left({ }^{\circ}\right)$ & \multicolumn{2}{|c|}{72.2366} & \multicolumn{2}{|c|}{-3.631} \\
\hline Velocity coefficient of helical motion $h l(\mathrm{~mm} / \mathrm{rad})$ & \multicolumn{2}{|c|}{0} & \multicolumn{2}{|c|}{0.766} \\
\hline
\end{tabular}

TABLE 5: Optimized machine tool parameters of the pinion.

\begin{tabular}{lcc}
\hline & $\begin{array}{c}\text { Levenberg-Marquard algorithm with trust region } \\
\text { strategy }\end{array}$ & $\begin{array}{c}\text { Sequential quadratic programming } \\
\text { algorithm }\end{array}$ \\
\hline Tilt angle $i\left(^{\circ}\right)$ & 14.641 & 15.049 \\
Swivel angle $j\left(^{\circ}\right)$ & -36.249 & -36.018 \\
Radial distance $s_{r 1}(\mathrm{~mm})$ & 117.968 & 117.721 \\
Center roll position $q_{1}\left({ }^{\circ}\right)$ & 65.626 & 65.626 \\
Work offset $e_{1}(\mathrm{~mm})$ & 29.100 & 28.880 \\
Machine center to back $x_{g 1}(\mathrm{~mm})$ & -0.138 & -0.117 \\
Sliding base $x_{b 1}(\mathrm{~mm})$ & 15.938 & 16.303 \\
Ratio of roll $m_{b 1}$ & 5.376 & 5.358 \\
Machine root angle $\gamma_{m 1}\left({ }^{\circ}\right)$ & -2.671 & -3.077 \\
Velocity coefficient of helical motion $h l$ & 0.516 & 0.595 \\
$(\mathrm{~mm} / \mathrm{rad})$ & & \\
\hline
\end{tabular}

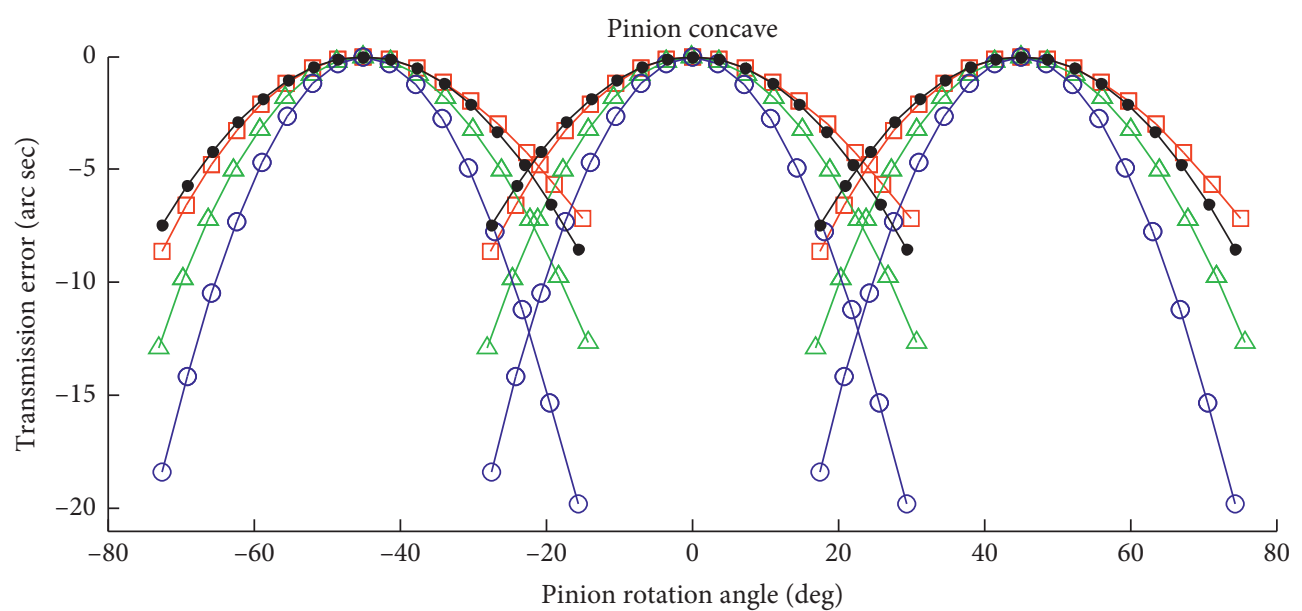

FIgURE 7: Initial curve, preset curve, optimized transmission error curve 1, and optimized transmission error curve 2 for the concave surfaces of the pinion.

and $7.6^{\prime \prime}$, i.e., $57.1 \%$ and $42.2 \%$, respectively. In summary, optimized transmission error curve 1 is consistent with the preset transmission error curve. Furthermore, the amplitudes of the meshing transition point and maximum transmission error are significantly reduced in comparison to the initial transmission error curve. These characteristics verify the effectiveness of the proposed method, which involves the precise, nonlinear optimization of the hypoid gear transmission error driven by the duplex helical method.

5.2. Loaded Tooth Contact Analysis. Because this study was based on the optimization of unloaded transmission errors and only the deviation of the contact point of the tooth surface is corrected, it was necessary to use loaded tooth contact analysis for the initial and optimized hypoid gear 


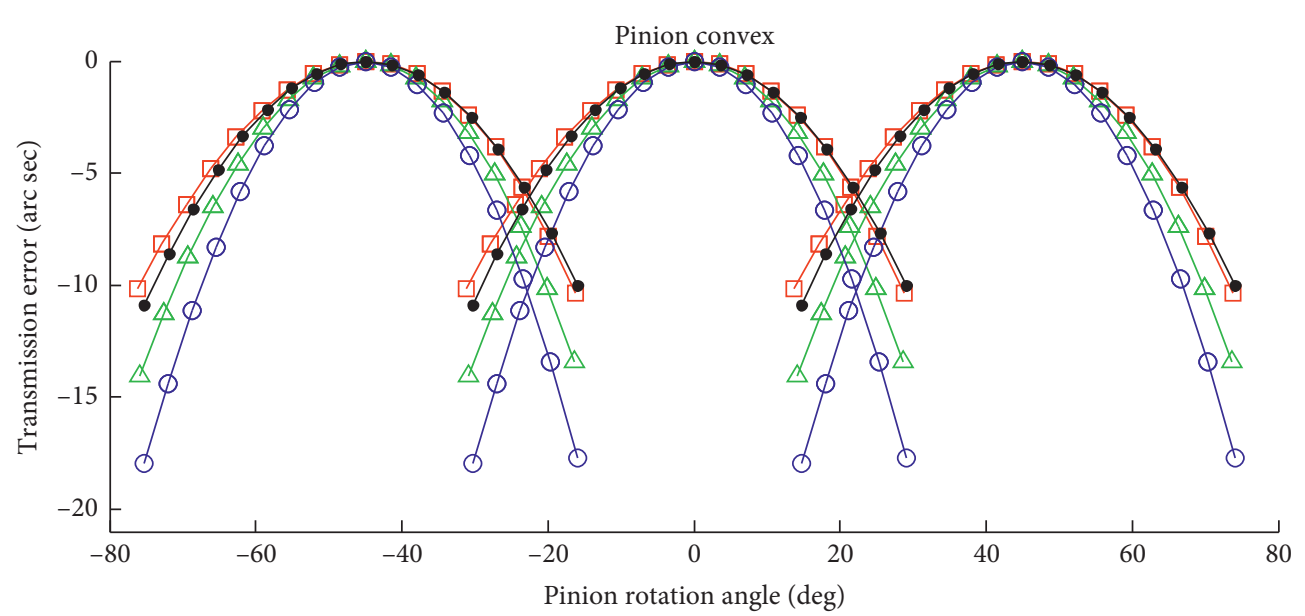

Figure 8: Initial curve, preset curve, optimized transmission error curve 1, and optimized transmission error curve 2 for the convex surfaces of the pinion.

TABLE 6: Maximum amplitude and amplitude of the meshing transition point for the transmission error.

\begin{tabular}{|c|c|c|c|c|}
\hline & \multicolumn{2}{|c|}{$\begin{array}{l}\text { Amplitude of meshing } \\
\text { transition point }\end{array}$} & \multicolumn{2}{|c|}{$\begin{array}{l}\text { Amplitude of maximum } \\
\text { transmission error }\end{array}$} \\
\hline & Concave & Convex & Concave & Convex \\
\hline Initial $\left({ }^{\prime \prime}\right)$ & 12.1 & 10.3 & 19.8 & 18.0 \\
\hline Preset $\left({ }^{\prime \prime}\right)$ & 5 & 6 & 8.6 & 10.9 \\
\hline Optimized transmission error curve $1\left(^{\prime \prime}\right)$ & 4.9 & 5.8 & 8.5 & 10.4 \\
\hline Optimized transmission error curve $2\left({ }^{\prime \prime}\right)$ & 7.6 & 7.9 & 12.9 & 14.1 \\
\hline
\end{tabular}

pairs to obtain the initial and optimized loaded contact pattern and loaded transmission errors. First, the tooth surfaces of the gear and pinion were solved according to the cutting mathematical model of the hypoid gear pair. Second, the TCA was used to obtain the installation position of the hypoid gear pair, to establish the assembly model of the hypoid gear pair through Pro/E software, and single teeth were cut on the pinion and the gear in the assembly model. Third, the single tooth assembly model was imported into Hypermesh software for 3D meshing. Finally, the single tooth assembly model with $3 \mathrm{D}$ mesh is imported into Abaqus software, and the single tooth model array is a five-tooth model; thus, the five-tooth model was subjected to loading tooth contact analysis based on the finite element method. For finite element analysis in Abaqus software, the density, elastic modulus, and Poisson's ratio were set to $7.85 \times 103 \mathrm{~kg} / \mathrm{m} 3$, $1.88 \times 105 \mathrm{MP}$, and 0.3 , respectively. In addition, the rotation speed of the pinion and the load torque of the gear were set to $1000 \mathrm{r} / \mathrm{min}$ and $200 \mathrm{~N} * \mathrm{~m}$, respectively. Figure 9 shows the finite element model of loaded tooth contact analysis of a hypoid gear pair with five pairs of teeth.

The loaded contact pattern was derived from a third tooth pair in the finite element model. Figures 10 and 11 show the loaded contact patterns for the concave and convex surface of the initial and optimized pinions, respectively. Figure 12 shows the initial and optimized loaded transmission error curves for the concave and convex surfaces of the pinion. The solid and dashed lines in Figure 12 represent the initial and optimized loaded transmission error curves, respectively.
Figures 10 and 11 show that the contact areas on the concave and convex surfaces of the initial and optimized hypoid gear pairs are the same. The maximum contact pressure for the concave surface of pinion is reduced from $333 \mathrm{MP}$ initially to $329 \mathrm{MP}$ after optimization. The maximum contact pressure for the convex surface of the pinion increased from $331 \mathrm{MP}$ initially to $344 \mathrm{MP}$ after optimized. As can be seen from Figures 7, 8, and 12, compared with the unloaded transmission error, the amplitudes of the initial and optimized loaded transmission error curves at the meshing transition point are reduced. For the concave surface, the amplitudes of the loaded transmission error curves of the initial and optimized pinions at the meshing transition point are $4 \mu \mathrm{m}$ and $8.9 \mu \mathrm{m}$, respectively. For the convex surface, the amplitudes of the loaded transmission error curves of the initial and optimized pinions at the meshing transition point are $4.6 \mu \mathrm{m}$ and $8.1 \mu \mathrm{m}$, respectively.

5.3. Contact Area Rolling Test of the Optimized Gear Pair. The $\mathrm{H} 650 \mathrm{C}$ spiral bevel gear milling machine produced by Haarang Kaishuai Precision Machinery Co., Ltd. was used to cut the gear pairs optimized by the Levenberg-Marquard algorithm with trust region strategy, and the contact area rolling test was carried out for the gear pairs after cutting. Figure 13 shows the cutting machine of the gear pair; Figure 14 shows the optimized hypoid gear pair that have been machined; and Figure 15 shows the optimized contact areas of the concave and convex surfaces of the pinion. 


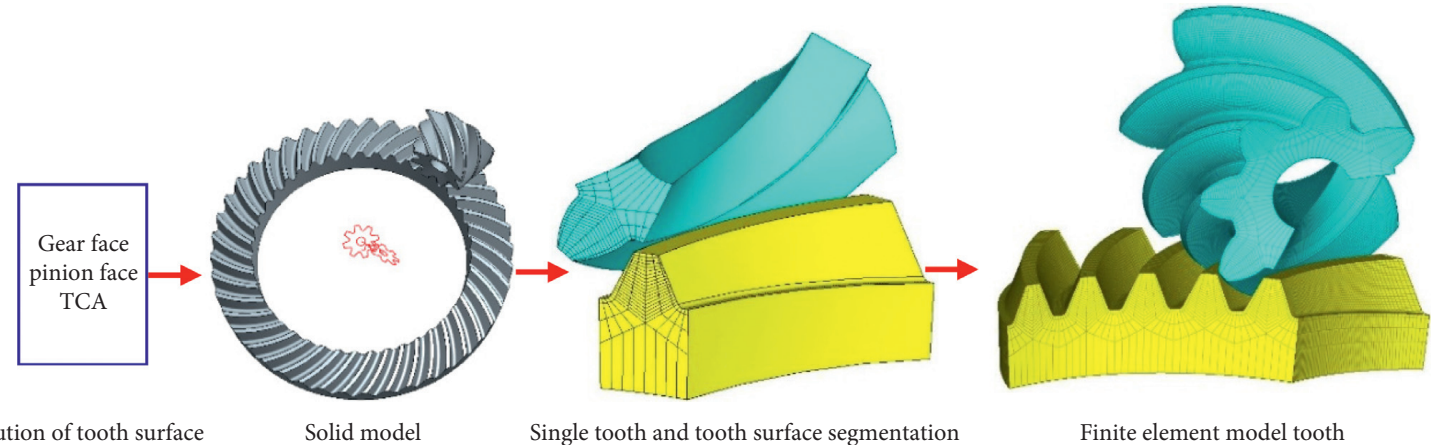

FIGURE 9: Finite element model of loaded tooth contact analysis of hypoid gear.

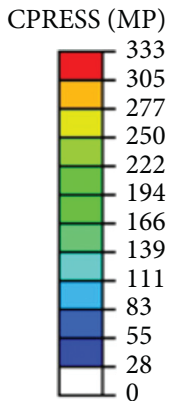

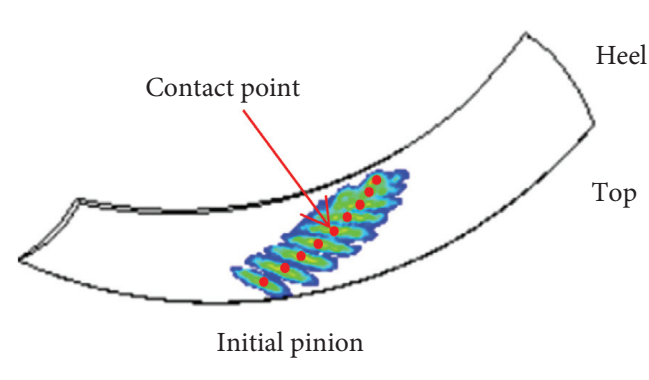

(a)
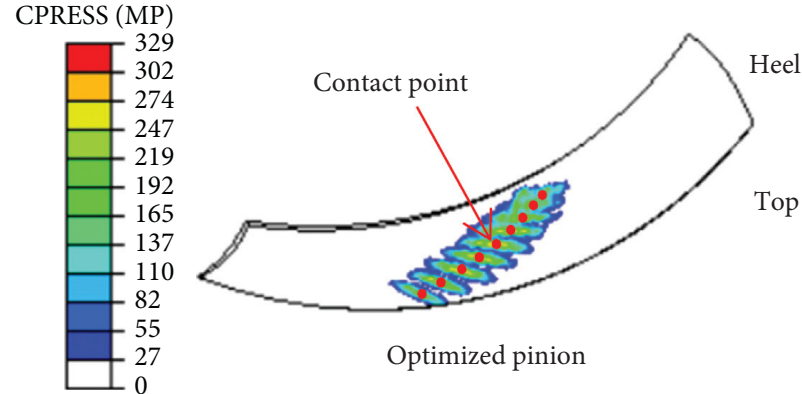

(b)

FIGURE 10: Loaded contact pattern for the concave surface of the initial and optimized pinion.

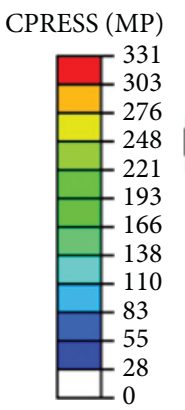

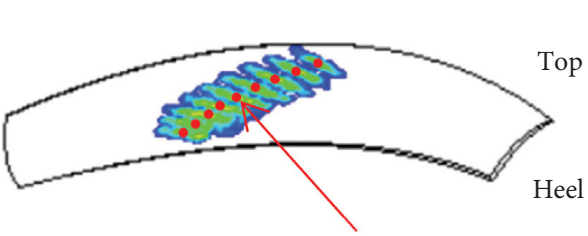

Contact point

Initial pinion

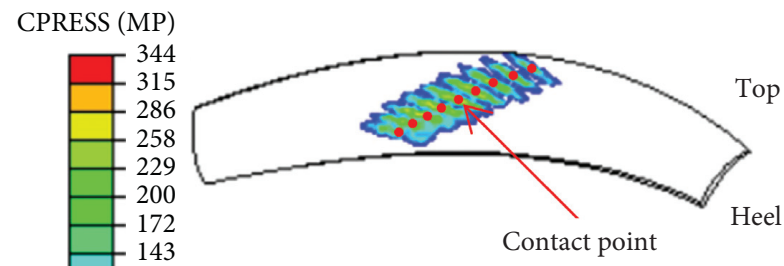

Optimized pinion

(a)

(b)

FIGURE 11: Loaded contact pattern for the convex surface of the initial and optimized pinion.

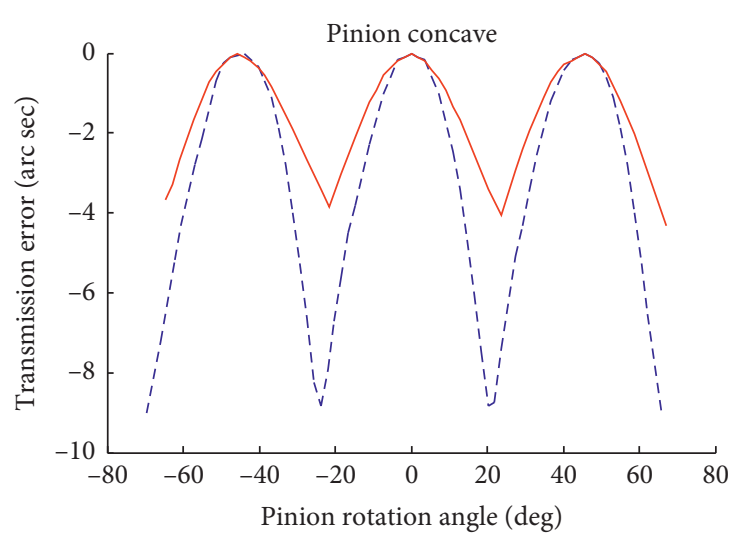

(a)

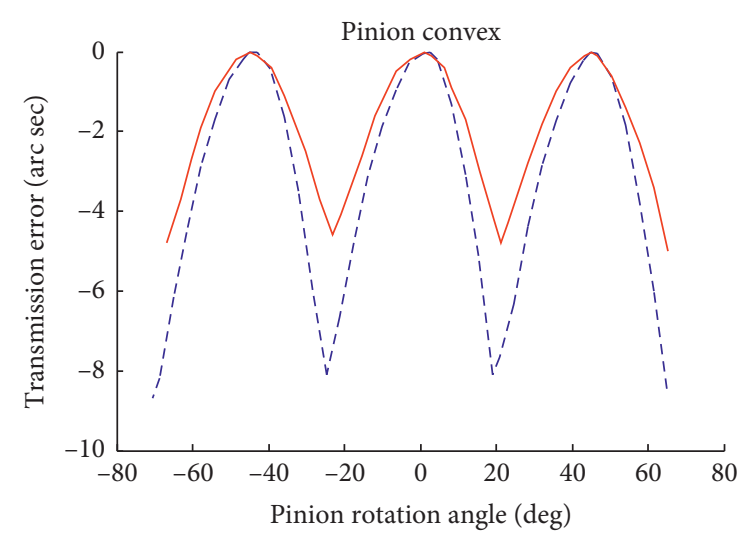

(b)

FIGURE 12: Loaded transmission error for the initial and optimized pinion. 


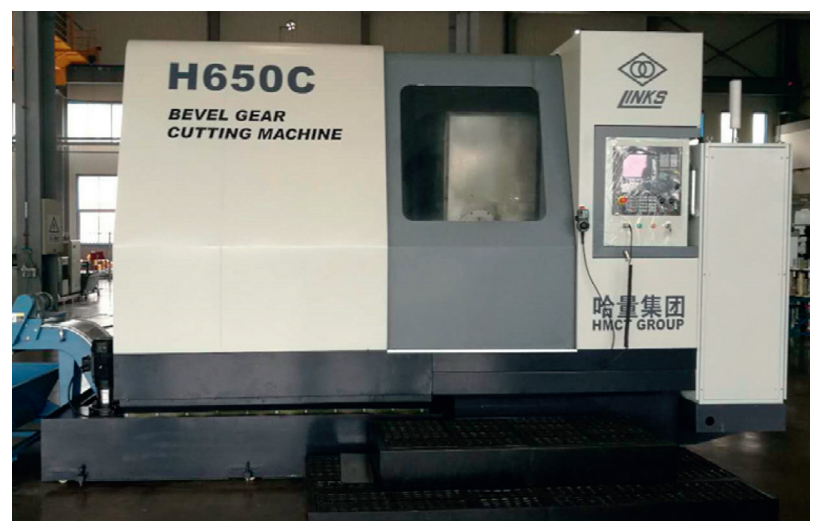

FIGURE 13: Cutting machine of the gear pair.

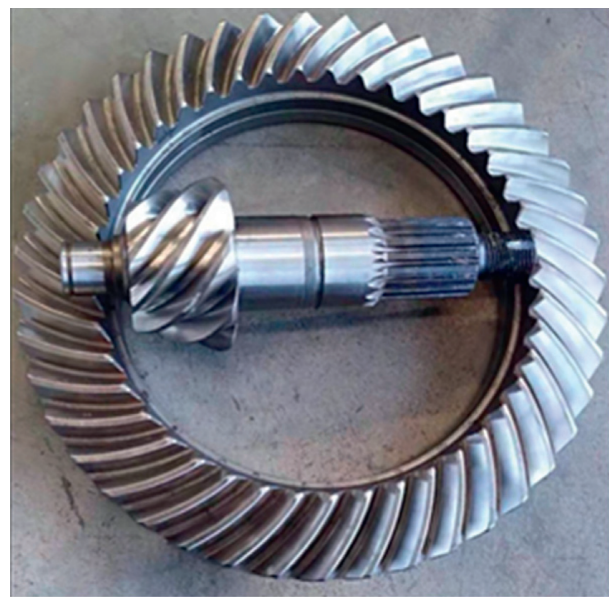

FIGURE 14: Optimized hypoid gear pair that has been machined.

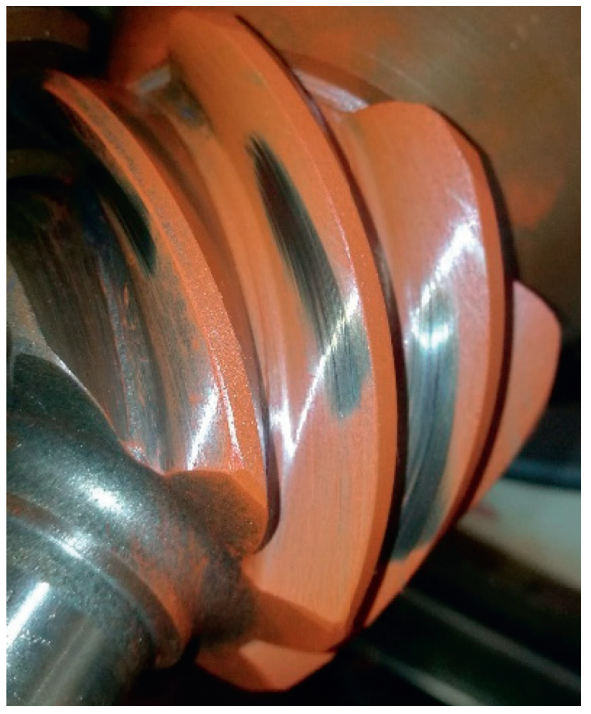

(a)

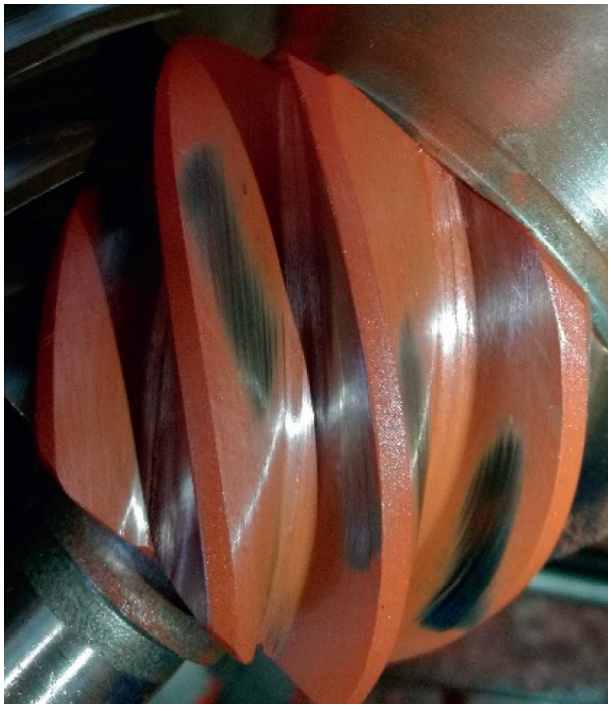

(b)

FIgURE 15: Optimized contact areas of the concave and convex surfaces of the pinion. (a) Concave. (b) Convex. 
Figure 15 shows that the rolling contact areas of the concave and convex surfaces of the pinion are good and are basically the same as the optimized loading contact areas in Figures 10 and 11. The contact area rolling test further shows that, after correcting the deviation of the tooth surface contact point to achieve high-precision optimization of the transmission error, the optimized tooth surface contact area can also meet the tooth surface design requirements. Therefore, for a hypoid gear machined by the duplex helical method, only the deviation of the tooth surface contact point needs to be corrected to obtain the corresponding machine tool parameters and, thereby, to obtain the required transmission error curve of the gear pair. Thus, it is not necessary to obtain the transmission error curve by cutting the tooth surface multiple times. The design and manufacturing efficiency of gear pair are improved. In addition, the proposed method can also be applied to the spiral bevel gear processed by the five-cut method.

\section{Conclusions}

This report proposed a transmission error optimization method for hypoid gears based on the duplex helical method, that is, only by correcting the deviations of the contact points between the target and initial pinion tooth surfaces, the transmission error of the concave and convex surfaces are optimized synchronously. The optimization algorithm used is the Levenberg-Marquard algorithm with trust region strategy, which was compared with the sequential quadratic programming algorithm. In terms of the consistency between the optimized and the preset transmission error curves, the Levenberg-Marquard algorithm with trust region strategy was better than the sequential quadratic programming algorithm. In addition, the transmission error curve optimized by the Levenberg-Marquard algorithm with trust region strategy was compared with the initial and preset transmission error curves, and the following conclusions were obtained.

(1) When comparing with the preset values, the amplitudes of the meshing transition points of the concave and convex surfaces of the optimized pinion differ by $0.1^{\prime \prime}$ and $0.2^{\prime \prime}$, i.e., $2 \%$ and $3.3 \%$, respectively. The amplitudes of the maximum transmission error of the concave and convex surfaces of the optimized pinion differ by $0.1^{\prime \prime}$ and $0.5^{\prime \prime}$, i.e., $1.2 \%$ and $4.6 \%$, respectively. When comparing with the initial values, the amplitudes of the meshing transition points of the concave and convex surfaces of the optimized pinion are reduced by $7.2^{\prime \prime}$ and $4.5^{\prime \prime}$, i.e., $59.5 \%$ and $43.7 \%$, respectively. The maximum transmission error amplitudes for the concave and convex surfaces of the optimized pinion are reduced by $11.3^{\prime \prime}$ and 7.6", i.e., $57.1 \%$ and $42.2 \%$, respectively. In other words, the optimized transmission error curve not only maintains the consistency with the preset transmission error curve but also exhibited reduced amplitudes of the mesh conversion point and maximum transmission error compared with those of the initial transmission error curve.

(2) The loaded contact areas on the concave and convex surfaces of the initial and optimized hypoid gear pairs were basically the same. The amplitudes of loaded transmission error curves at the meshing transition points of the concave and convex surfaces of the initial and optimized pinion were reduced. The effectiveness of the nonlinear optimization method for the transmission error of the hypoid gear was further verified by loaded tooth surface contact analysis. However, the maximum contact pressure of the concave surfaces of the pinions increased from $331 \mathrm{MP}$ initially to $344 \mathrm{MP}$ after optimization. Therefore, factors such as contact pressure and transmission efficiency need to be considered in research.

(3) The tooth surface cutting test revealed that the rolling contact areas of the concave and convex surfaces of the optimized pinion were basically the same as the loading contact areas obtained by the finite element method. That is, the optimized tooth surface contact area was good, and there is no edge contact problem, which further supports the effectiveness of the proposed transmission error curve optimization method.

\section{Nomenclature}

$a_{p}: \quad$ Blade angle of the pinion head cutter

$r_{c 1}: \quad$ Cutter point radius of pinion

$\theta_{p}, \theta_{\mathrm{g}}, u_{p}$, Surface parameters of the pinion and gear head

$u_{g}: \quad$ cutters Gaussian coordinates

$s_{r 1}$ : $\quad$ Radial distance of head cutter of the pinion

$q_{1}$ : $\quad$ Center roll position of pinion

$\gamma_{m 1}$ : Machine root angle of pinion

$x_{g 1}$ : Machine center to cross point of pinion

$i, j: \quad \quad$ Tilt angle and swivel angle

$\varphi$ : $\quad$ Angle of rotation of the cradle in the process for generating the pinion

$m_{b 1}$ : $\quad$ Rolling ratio of the pinion

$e_{1}$ : $\quad$ Work offset of the cutting pinion

$x_{b 1}$ : $\quad$ Sliding base for the pinion

hl: $\quad$ Velocity coefficient of helical motion

$w^{(1)}, w^{(2)}$ : Angular velocity of the pinion and gear in the process of meshing

$\Sigma^{(0)}, \Sigma^{*}: \quad$ Initial pinion tooth surface and target pinion tooth surface

E: $\quad$ Pinion offset in a gear pair

$\Gamma$ : Shaft angle

$\mathbf{r}_{t}^{(0)} \mathbf{n}_{t}^{(0)}$ : Radial vector and unit normal vector of the contact point for the initial pinion

$L_{t}, R_{t}$ : Contact point of the initial pinion on the

rotating projection surface

$\psi_{1}^{(0)} \psi_{2}^{(0)}$ : Initial rotation angle of the pinion and gear in the process of meshing

$\psi_{1}, \psi_{2}$ : Rotation angle of the pinion and gear in the process of meshing 
$\delta_{\mathrm{te}}: \quad \quad \quad \quad \quad \quad$ The amplitude of the meshing transition point

$z_{1}, z_{2}$ : Number of teeth of the pinion and gear

$\triangle \psi_{2}\left(\psi_{1}\right):$ Function of the transmission errors

$\mathbf{r}_{t}^{*} \mathbf{n}_{t}^{*}$ : Radial vector and unit normal vector of the contact point of the target pinion

$M_{1 p}, L_{1 p}$ : Transformation matrix from the coordinate system $S_{p}$ to system $S_{1}$.

\section{Data Availability}

The data used to support the findings of this study are available from the corresponding author upon request.

\section{Conflicts of Interest}

The authors declare that they have no conflicts of interest.

\section{Acknowledgments}

This work was substantially supported by the National Natural Sciences Foundation of China (Grant nos. 52075552, 51575533, 51805555, and 11662004).

\section{References}

[1] A. Artoni, M. Gabiccini, and M. Guiggiani, "Nonlinear identification of machine settings for flank form modifications in hypoid gears," Journal of Mechanical Design, vol. 130, no. 6, p. 112602, 2008.

[2] A. Artoni, A. Bracci, M. Gabiccini, and M. Guiggiani, "Optimization of the loaded contact pattern in hypoid gears by automatic topography modification," Journal of Mechanical Design, vol. 139, no. 5, Article ID 011008, 2009.

[3] H. J. Stadtfeld, Gleason Bevel Gear Technology, The Gleason Works, New York, NY, USA, 1994.

[4] I. GonzalezPerez, A. Fuentes, and K. Hayasaka, "Computerized design and tooth contact analysis of spiral bevel gears generated by the duplex helical method," in Proceedings of the ASME 2011 International Design Engineering Technical Conferences and Computers and Information in Engineering Conference, pp. 149-158, Washington, DC, USA, August 2011.

[5] Y.-b. Zhuo, X.-y. Xiang, X.-j. Zhou, H.-l. Lv, and G.-y. Teng, "A method for the global optimization of the tooth contact pattern and transmission error of spiral bevel and hypoid gears," Journal of Zhejiang University-SCIENCE A, vol. 18, no. 5, pp. 377-392, 2017.

[6] H. Ding, J. Tang, J. Zhong, and Z. Zhou, "A hybrid modification approach of machine-tool setting considering high tooth contact performance in spiral bevel and hypoid gears," Journal of Manufacturing Systems, vol. 41, pp. 228-238, 2016.

[7] F. L. Litvin, A. Fuentes, and K. Hayasaka, "Design, manufacture, stress analysis, and experimental tests of low-noise high endurance spiral bevel gears," Mechanism and Machine Theory, vol. 41, no. 1, pp. 83-118, 2006.

[8] V. V. Simon, "Design and manufacture of spiral bevel gears with reduced transmission errors," Journal of Mechanical Design, vol. 131, no. 4, Article ID 041007, 2009.

[9] X. Wang, Z. G. Fang, Y. M. Mu, J. F. Du, and Y. F. Cui, "Optimization design of loaded transmission error for HGT hypoid gear drives," Journal of Vibration and Shock, vol. 36, no. 8, pp. 34-40, 2017.
[10] Q. Wang, C. Zhou, L. Gui, and Z. Fan, "Optimization of the loaded contact pattern of spiral bevel and hypoid gears based on a kriging model," Mechanism and Machine Theory, vol. 122, pp. 432-449, 2018.

[11] G. L. Liu and H. W. Fan, "A better active design for optimizing grinding parameters of aviation spiral bevel gears," Journal of Northwestern Polytechnical University, vol. 29, no. 3, pp. 394-399, 2011.

[12] A. Artoni, M. Kolivand, and A. Kahraman, "An ease-off based optimization of the loaded transmission error of hypoid gears," Journal of Mechanical Design, vol. 132, no. 1, Article ID 011010, 2010.

[13] J. Astoul, E. Mermoz, M. Sartor, J. M. Linares, and A. Bernard, "New methodology to reduce the transmission error of the spiral bevel gears," CIRP Annals, vol. 63, no. 1, pp. 165-168, 2014.

[14] T. J. Krenzer, Computer Aided Corrective Machine Settings for Manufacturing Bevel and Hypoid Gear Sets, American Gear Manufacturers Association, Washington, DC, USA, 1984.

[15] F. L. Litvin, C. Kuan, J. C. Wang, and R. F. Handschuh, "Minimization of deviation of gear real tooth surface determined by coordinate measurements," Journal of Mechanical Design, vol. 115, no. 4, pp. 955-1001, 1993.

[16] H. J. Stadtfeid, Handbook of Bevel and Hypoid Gears, Rochester Institute of Technology, New York, NY, USA, 1993.

[17] C.-Y. Lin, C.-B. Tsay, and Z.-H. Fong, "Computer-aided manufacturing of spiral bevel and hypoid gears with minimum surface-deviation," Mechanism and Machine Theory, vol. 33, no. 6, pp. 785-803, 1998.

[18] C.-Y. Lin, C.-B. Tsay, and Z.-H. Fong, "Computer-aided manufacturing of spiral bevel and hypoid gears by applying optimization techniques," Journal of Materials Processing Technology, vol. 114, no. 1, pp. 22-35, 2001.

[19] Y.-P. Shih, "A novel ease-off flank modification methodology for spiral bevel and hypoid gears," Mechanism and Machine Theory, vol. 45, no. 8, pp. 1108-1124, 2010.

[20] H. Ding, J. Y. Tang, and J. Zhong, "On the identification of machine settings for gear surface topography corrections," Journal of Mechanical Design, vol. 134, no. 4, Article ID 041004, 2012.

[21] H. Ding, J. Tang, and J. Zhong, "An accurate model of highperformance manufacturing spiral bevel and hypoid gears based on machine setting modification," Journal of Manufacturing Systems, vol. 41, pp. 111-119, 2016.

[22] S. Peng, H. Ding, and J. Tang, "Accurate numerical computation of loaded tooth surface contact pressure and stress distributions for spiral bevel gears by considering timevarying meshing characteristics," Advances in Engineering Software, vol. 135, p. 102683, 2019.

[23] M. Vivet, D. Mundo, T. Tamarozzi, and W. Desmet, "An analytical model for accurate and numerically efficient tooth contact analysis under load, applied to face-milled spiral bevel gears," Mechanism and Machine Theory, vol. 130, pp. 137-156, 2018.

[24] A. Fuentes-Aznar, R. Ruiz-Orzaez, and I. Gonzalez-Perez, "Numerical approach for determination of rough-cutting machine-tool settings for fixed-setting face-milled spiral bevel gears," Mechanism and Machine Theory, vol. 112, pp. 22-42, 2017.

[25] M. Kolivand and A. Kahraman, "An ease-off based method for loaded tooth contact analysis of hypoid gears having local and global surface deviations," Journal of Mechanical Design, vol. 132, no. 7, Article ID 071004, 2010. 
[26] M. J. D. Powell, A Hybrid Method for Nonlinear Equations, Gordon \& Breach, London, UK, 1970.

[27] J. E. Dennis and H. H. W. Mei, "Two new unconstrained optimization algorithms which use function and gradient values," Journal of Optimization Theory and Applications, vol. 28, no. 4, pp. 453-482, 1979.

[28] D. W. Marquardt, "An algorithm for least-squares estimation of nonlinear parameters," Journal of the Society for Industrial and Applied Mathematics, vol. 11, no. 2, pp. 431-441, 1963. 